\title{
Soziale Sicherheit und Staatsfinanzen
}

\author{
von Alfred Radner *
}

\section{SUMMARY : SOCIAL SECURITY AND GOVERNMENT FINANCE}

A recapitulation, a piecing-together of the knowledge fragmented by the specialization necessary today, is more vital than ever.

Developments in the field of social policy are dramatic and have multiple repercussions on almost every sphere of community life, which naturally includes the general and special sectors of the economy and finance.

Government finance performs specific functions which closely affect the social policy sphere ; more important even than its re-direction of market economy processes, it must bring into better effect an equitable apportioning - before or after taxes - of the economic yield and state relief services to the various income or other sociologically relevant groups. Social insurance falls within these functions of the state economy, as on the one hand it lowers wages by absorbing contributions and on the other constitutes or raises wages by concession of benefits within these two spheres of influence of the financial policy sector.

The flows of finance generated by social insurance through the public budgets become stronger from year to year. This has no mean influence on the redistribution of goods and services within the economy in both destination and extent and in no way facilitates fiscal handling; in consequence, the shift of the social yield is becoming increasingly economic in character. Receipts and outlay for social security have gathered such tremendous momentum that they determine to a great extent the course of the whole economy.

Re-distribution as it pertains to social security differs from other measures of social policy which aim at wage re-apportionment (e.g. progressive income taxation, tax privileges for families, old and economically weak persons, family allocations, etc.) in that it is not, essentially, a shift from higher to lower income brackets, but seeks to meet specific income requirements, such as illness, invalidity, old age, unemployment, partial or total incapacity to work through working accidents, etc.

To achieve an end result as permanent and comprehensive as possible, the individual component measures must be synchronized. Thus the re-apportioning of benefits must be co-ordinated so as to ensure decent (minimum) wages; contributions and fiscal policies must be consequently adapted.

Therefore, each measure must be assessed in the light of those that are parallel to it, overlap it, or even run contrary to it. The relevant amounts must then be co-ordinated to achieve a judicious overall end effect.

* University of Linz, Austria. This paper is part of a research which has been granted an "Ernst Meyer" Scholarship of the Geneva Association. 


\section{Einleitung}

Das soziale Sicherheitsbedürfnis wächst zusehends in dem Maße, als der einzelne Staatsbürger Bewußtsein darüber erlangt, daß es immer schwerer wird, sich in der immer unübersichtlicher und verwirrender werdenden Gesellschaftsordnung zurecht $\mathrm{zu}$ finden. Eine Vielfalt vom Komponenten wirken auf ihn ein, die er nicht aus eigener Kraft beeinflussen kann. Es bedarf für Entscheidungen in immer weiteren Bereichen des gesellschaftlichen Zusammenlebens immer mehr der Meinungsbildung in Personenmehrheiten.

Je weiter aber der Kreis der demokratischen Mitentscheidung gezogen wird und je mehr Staatsbürger in vielfältigen Belangen mitentscheiden, umso mehr werden die Dispositionsmöglichkeiten des Einzelnen eingeschränkt. Dies zeigt sich etwa bei der Diskussion um die betriebliche Mitbestimmung, nach der der Entscheidungsspielraum in den Unternehmen modifiziert werden soll, ebenso wie bei den Aktivitäten der Bürgerinitiativen, die nunmehr auch zwischen den Wahlen die gewählten politischen Mandatare - welche die Interessensvertretungen ihrer Wähler bisher nach eigener Verantwortlichkeit für sich in Anspruch nahmen - mit der Wählermeinung konfrontieren.

Die Zunahme an Möglichkeiten der Mitgestaltung läßt jedoch den Einzelnen die Notwendigkeit immer deutlicher erkennen, daß er seine Interessen nur dann durchsetzen kann, wenn er eine Unterstützung durch eine Personenvielfalt findet. Darin offenbart sich aber ein Paradoxon unserer Gesellschaft, nämlich in der Form, daß den größeren Möglichkeiten an Mitentscheidung der immer kleiner werdende Bereich der durch eigene Entscheidung zu gestaltende Spielraum gegenübersteht.

Aus der konsequenten Fortführung dieser Entwicklung ergibt sich aber, daß der Einzelne von der Gesellschaft immer mehr Sicherungsleistungen in immer weiteren Bereichen erwartet und zu erwarten hat. Dieses kollektive Sicherheitsstreben soll unter anderem die individuelle Lebensangst absorbieren. Der Mensch ist ein "ens soziale", der früher in der Großfamilie, der Sippe, seine Geborgenheit fand und heute in Zeiten der Kleinfamilie, die dem Einzelnen zwar mehr persönliche Freiheit vermittelt, jedoch anfälliger gegen die Wechselfälle des Lebens macht, diesen Schutz der Großfamilie in der Gesamtgesellschaft sucht.

Diese Schutzaufgaben der Gesamtgesellschaft gegen die Wechselfälle des Lebens übernahm in einem sehr hoch entwickelten Ausmaß die gesetzliche Sozialversicherung mit ihren Zweigen der Kranken-, Unfall- und Pensionsversicherung sowie der Arbeitslosenversicherung. Für viele ist dieser Schutz der Sozialversicherung schon so sehr zu einer Selbstverständlichkeit geworden, daß der Gedanke der kollektiven Selbsthilfe kaum mehr ins Bewußtsein dringt. Doch gerade die ständig steigenden Aufgaben der Sozialversicherung mit dem zwangsläufig verbundenen zusätzlichen Bedarf an Mitteln erfordern ein hohes $\mathrm{Maß}$ an Verständnis aller Staatsbürger für diese Einrichtungen und deren gesellschaftliche Mittlerfunktion zum Mitbürger.

Am deutlichsten tritt diese im Rahmen der Einkommensumverteilung zutage, wobei diese vor allem im wirtschaftlichen Kreislauf sehr nachhaltige Spuren hinterläßt und richtungsweisende Impulse setzt.

Die zunehmende finanzwirtschaftliche Bedeutung der Sozialversicherung zeigt sich in einer Verlagerung der Sozialleistungen des Bundes auf die Sozialversicherungsträger. 
So sind die eigentlichen Versorgungs- und Fürsorgeleistungen des Bundes stark zurückgegangen, während die Ausgaben der Sozialversicherungsträger ständig steigen.1

\section{Die Elnkommenumvertellung durch die Sozialversicherung im Kreislaufsystem der Finanzen}

\subsection{Spezifische Umverteilung durch die Sozialversicherung}

Zum Unterschied von anderen sozialpolitischen Maßnahmen, die der Umverteilung von Einkommen dienen, z.B. progressive Einkommensbesteuerungen, Steuerbegünstigungen für Familien, älteren Personen, wirtschaftlich Schwachen, Familienbeihilfen usw. handelt es sich bei der Redistribution durch die Sozialversicherung im wesentlichen nicht so sehr um die Verschiebung von höheren zu niedrigeren Einkommensgruppen, sondern um die Deckung spezifischer Einkommensbedürfnisse, so etwa wegen Krankheit, Invalidität, Alter, Arbeitslosigkeit, teilweise oder gänzlicher Ausfall der Arbeitskraft durch einen Arbeitsunfall usw. ${ }^{2}$

Seitdem die Selbstversorgung mit Naturalien vom Geldwirtschaftssystem abgelöst wurde, ist für die Erlangung von lebensnotwendigen Gütern und Dienstleistungen wie Nahrung, Kleidung, Wohnung, der Besitz von Geld erforderlich. Die dafür notwendigen Geldmittel werden durch Zurverfügungstellung der einzelnen, vom Markt verwertbaren Produktionsfaktoren in Form von Kapital, Grund und Boden oder Arbeitskraft geleistet, wie es eben in einer arbeitsteiligen Wirtschaft nicht anders möglich ist. Die markantesten Merkmale einer arbeitsteiligen Wirtschaft moderner Prägung sind nämlich die Aufspaltung der wirtschaftlichen "Zellen" in Produktions- und Konsumgemeinschaften und zwar Unternehmungen und Haushalte. ${ }^{3}$

Die einzelnen Personen - die zweckmäßigerweise für solche Untersuchungen in Haushalte zusammengefaßt werden, weil in diesem Bereich die interfamiliäre Umverteilung zwischen Einkommensbezieher (in der Regel der Familienvater) und den Kindern sowie den nichtberufstätigen Müttern erfolgt - , stellen den Unternehmungen Produktionsfaktoren zur Verfügung. In den Unternehmungen werden diese Faktoren im Produktionsprozeß kombiniert und transferiert zu Gütern und Dienstleistungen, die über die marktwirtschaftliche Verteilung zur Bedürfnisbefriedigung an die Haushalte zurückfließen (Güterseite des Kreislaufes). Auf der Gegenseite des Kreislaufes fließen, den Produktionsfaktoren entgegengesetzt, Entgelte als Einkommen den Haushalten zu, so wie den Gütern und Dienstleistungen entgegengesetzt von den Haushalten zu den Unternehmungen die Entgelte in Form von Umsätzen und Erlösen fließen. ${ }^{4}$

Nun gibt es aber in der großen Gemeinschaft einer Volkswirtschaft eine Vielzahl von Personen, die über keine oder keine ausreichende Menge von marktwirtschaftlich verwertbaren Produktionsfaktoren verfügen. Das sind vor allem die Kranken, Gebrechlichen, Arbeitslosen, Alten, Invaliden, Waisen usw. Da bei diesem Personenkreis der Einsatz des Produktionsfaktors "Arbeit" naturgemäß ausfällt, müßte diese, um dem

1 Wilhelm Weber : Österreichische Finanzpolitik $1945-1961$, Wien 1962, Seite 121.

2 Wilhelm Weber, a.a.O., Seite 115.

3 Heinz Haller: Finanzpolitik, Tübingen-Zürich 1961, Seite 9.

4 Paul A. Samuelson: Volkswirtschaftslehre, 3. Aufiage, Köln 1964, Band 1, Seite 249. 
marktgemäßen Verteilungsprozeß zu entsprechen, ausreichend mit den Produktionsfaktoren "Kapital" und "Grund und Boden" versorgt werden. Da jedoch die technische Durchführbarkeit einer solchen Vermögensbildung und -verteilung nicht gegeben ist, muß die Versorgung der nichtproduktiven Personen durch die am Leistungsprozeß Beteiligten erfolgen. Dies geschieht durch die Umverteilung - im Wege von Beiträgen und Steuern - vom Einkommen der Erwerbstätigen auf die Nichterwerbstätigen.

Das System der sozialen Sicherheit hat - was bei diesem Modell leicht übersehen werden könnte - aber nicht allein "Lohnersatzfunktionen" sondern auch weitgehende und umfassendere Aufgaben wie z.B. die Sachleistungen der Krankenversicherung (Ärztliche Hilfe, Heilmittel, Heilbehelfe usw.). Diese Leistungen haben eine Ausgleichsfunktion im Nachfrage-Preissystem und wirken daher regulierend auf die Einkommenund Ausgabenseite. ${ }^{5}$ Dies wirkt sich so aus, daß auf diese Weise auch jeder Erwerbstätige ein Stück zusätzlichen Ertrages seiner produktiven Leistung in Form von Sachleistungen erhält.

\subsection{Einkommensredistribution über die Staatseinnahmen}

In der privaten Produktions- und Tauschwirtschaft führt der Marktprozeß zu einer "funktionellen" und "personellen" Einkommensverteilung. Die funktionelle Einkommensverteilung hängt von der Verteilung und der Ertragsfähigkeit der Produktionsfaktoren $a b$, die personelle dagegen von der Art und Menge der Produktionsfaktoren, über die die einzelnen Personen (Haushalte) verfügen. ${ }^{6}$

Da jedoch die Produktionsfaktoren innerhalb einer Volkswirtschaft ungleich verteilt sind, ergeben sich ungleiche Einkommensverteilungen, die nach sozialmarktwirtschaftlichen Grundsätzen ausgeglichen werden müssen. Für diesen Ausgleich sind die wechselweisen Zusammenhänge zwischen Sozialversicherung und Budgetausgaben sowie Steuereinnahmen, wie dies im speziellen auch von Musgrave ${ }^{7}$ dargestellt worden ist, von großer Wichtigkeit.

\section{A) Einkommensverteilung über Steuern}

Die Einkommensverteilung über die Steuern trifft im wesentlichen alle. So werden Personen mit geringem Einkommen und solche, die Angehörige ohne eigenes Einkommen $\mathrm{zu}$ versorgen haben, einkommensteuermäßig geringer besteuert als andere Personen. Es wird daher auf die objektive Bedürfnissituation der Einkommensempfänger Rücksicht genommen. Diese Korrektur über die staatliche Finanzwirtschaft ist auf eine gerechtere Einkommensverteilung gerichtet. Es soll dem Familienerhalter, der für Angehörige, die nicht am Erwerbsprozeß teilnehmen können, ein höherer Grad der Bedürfnisbefriedigung ermöglicht werden.

Neben dem Steuerabzug tritt eine Einkommensverminderung der Erwerbstätigen auch durch den Abzug von Beiträgen zur Sozialversicherung ein. Dies ist ein interner Umverteilungsproze $ß$ hinsichtlich des betroffenen Personenkreises zugunsten einkommensschwächerer Gruppen, somit ein interpersoneller Ausgleich. Im Gegensatz zur Ein-

5 Dieter Schäfer : Soziale Schäden, soziale Kosten und soziale Sicherung, Sozialpolitische Schriften, Heft 29, Duncker \& Humblot, Berlin 1972, Seite 271 k.

6 Heinz Haller : Finanzpolitik, Tübingen-Zürich 1961, Seite 195.

7 Richard A. Musgrave, Finanztheorie, Tübingen 1969, 2. Auflage. 
kommensbesteuerung wird jedoch auf den Familienstand nicht Rücksicht genommen. Da jedoch in der Regel jeder Haushalt in bestimmten Zeiten für Angehörige ohne Einkommen $\mathrm{zu}$ sorgen hat und $\mathrm{zu}$ bestimmten Zeiten nicht, ergibt sich ein Ausgleich über größere Zeiträume hinweg, somit spricht man auch von einem intertemporalen Ausgleich. Daneben gibt es noch den interorganisatorischen Ausgleich durch den beim Hauptverband der österreichischen Sozialversicherungsträger errichteten Ausgleichsfonds der Krankenversicherungsträger ( $\$$ 447a ff. ASVG), die gesetzliche Mittelübertragung von der Unfallversicherung zur Pensionsversicherung und zur Krankenversicherung (Ersatzansprüche/Entgeltfortzahlung), sowie die gemeinsame Kostentragung von Krankenversicherung und Pensionsversicherung bei Rehabilitationsmaßnahmen.

B) Einkommensverteilung durch Sozialtransfers

Die zweite Seite des Einkommensausgleiches sind die Sozialversicherungsleistungen, die im Rahmen von Sozialtransfers, gleichgültig ob Bar- oder Sachleistungen, erbracht werden. Sie stellen beim Leistungsempfänger entweder überhaupt das Haupteinkommen dar oder treten $\mathrm{zu}$ einem verminderten Einkommen hinzu oder ersparen Ausgaben aus dem eigenen Einkommen, z.B. durch die Abdeckung der Kosten für die ärztliche Behandlung durch die Krankenversicherung. Sie führen daher bei den Versicherten, die Beiträge (und Steuern) entrichten, sowie keine Leistungen beziehen, zu einer Einkommensverminderung und bei den beitrags- und/oder steuerzahlenden Leistungsempfängern zu einer Einkommensbildung - oder zumindest zu einer Einkommensaufstockung oder Ausgabenersparnis. Insoweit wird dadurch ein Einkommensausgleich erwirkt.

Dieser Einkommensausgleich ist vertikaler Natur, also umfaßt eine Umverteilung von höheren auf niedrigere Einkommen, 8 wenn bei einem Sozialversicherungssystem mit Bundeszuschüssen das Steuerverhältnis eher die direkten Steuern (progressiv) bevorzugt. Aber auch durch die Sozialversicherungsbeiträge, die bei den Einkommen innerhalb der Höchstbeitragsgrundlage erhoben werden, führen, begünstigt durch die Rahmenbreite dieser Höchstbeitragsgrundlagen und deren alljährliche Anhebung auf Grund der Richtzahl nach dem Pensionsanpassungsgesetz, zu einem vertikalen Einkommensausgleich innerhalb der Versicherten. Neben dem vertikalen Einkommensausgleich findet aber auch ein horizontaler statt und zwar durch die Sozialversicherungsbeiträge, die Versicherte mit gleichen Beitragsgrundlagen, z.B. etwa der gleichen Lohnstufe, entrichten. Durch diesen horizontalen Einkommensausgleich werden jene Versicherten begünstigt, die z.B. durch einen schlechteren Gesundheitszustand genötigt sind, mehr Leistungen der Krankenversicherung in Anspruch zu nehmen. Da aber auch die Versicherten neben den Sozialversicherungsbeiträgen Steuern entrichten und ein Teil der Steuern auf die Güterpreise weitergewälzt wird, verstärkt sich der vorerst vertikale Umverteilungseffekt von höheren $\mathrm{zu}$ niedrigeren Einkommen teilweise auch zugunsten einer horizontalen Umverteilung.

C) Sachleistungstransfers

Bei Sachleistungen (z.B. Krankenbehandlung, Arzneimittel, Kuraufenthalte usw.) ist $\mathrm{zu}$ bemerken, daß diese Leistungen Einkommen darstellen, die vom Sozialversiche-

8 Wilhelm Weber: "Wachstumseffekte der Staatsausgaben", in : Finanztheorie, herausgegeben von Horst Claus Recktenwald, Köln-Berlin 1969, Seite 315. 
rungsträger in Form von Gütern und Diensten gekauft und den Empfängern in natura übertragen werden. Während die Empfänger von Bar-Übertragungseinkommen ihre Kaufentscheidung selbst treffen können, behält sich der Staat bzw. der Versicherungsträger bei den Sachleistungen - aus Gründen, auf die an dieser Stelle nicht eingegangen werden braucht - die Kaufentscheidung vor.

Im Sinne der volkswirtschaftlichen Gesamtrechnung sind Sachleistungen als bereits ausgegebene Bestandteile des Aggregates persönlich verfügbarer Einkommen anzusehen, denen ein gleich großer Konsumaufwand der Empfänger gegenübersteht. So werden z.B. die Arzneimittel aus Apotheken als Sachleistungen zur sozialen Sicherheit zu dem entsprechenden Marktpreis bzw. zu einem begünstigten Abgabenpreis gekauft. Durch die Rückvergütung der damit zusammenhängenden Umsatzsteuer (Mehrwertsteuer) an den Sozialversicherungsträger kommt diese Verminderung des "Marktpreises" dem Versicherten als Einkommensvorteil gegenüber einem Nichtversicherten zu, der ja diese Arzneien zum Marktpreis einschließlich der Umsatzsteuer erwerben muß. Nach den Empfehlungen der OECD ${ }^{9}$ sind zwar die Sachleistungen bei Einrichtungen zur sozialen Sicherung als Staatsverbrauch anzusehen, da die öffentliche Hand über Art und Höhe des Kaufes entscheidet, doch können diese Sachleistungen ebenso wie die Barleistungen als staatliche Einkommensübertragung dem Einkommen der Leistungsempfänger zugeordnet werden, da letztlich entscheidend ist, wem die Leistung als Einkommensvorteil zugerechnet werden kann.

\subsection{Herstellung und Erhaltung des Verteilungsgleichgewichtes}

Von sehr wesentlicher Bedeutung für die Herstellung und Erhaltung des sozialen Gleichgewichtes ist sowohl die primäre Verteilung der Einkommen, d.i. die Einkommenszuordnung auf Grund der Mitwirkung am Produktionsproze $\beta$ als auch die sekundäre Einkommensverteilung unter der Berücksichtigung der Einkommensabzüge sowie der Zuordnung von Transfereinkommen.

Es ist vor allem der ökonomische Verteilungsprozeß, dessen Ablauf durch sozialpolitische Maßnahmen im Rahmen der Sozialversicherung berichtigt werden soll. Die Normen hinsichtlich der Verteilungsproportionen und der Zurechnungsformeln unterliegen ständigen Anpassungsprozessen und sind ein supplementäres Instrument im Rahmen eines relativen freien Spieles der Kräfte in der Erwerbswirtschaft, ${ }^{10}$ die zugleich eine Verteilungswirtschaft ist.

Die Umverteilung erfolgt zwischen primären Einkommensbeziehern, die ein Markteinkommen beziehen und Nicht-Mehr-Leistenden (vertikal), sowie zwischen den Beziehern höherer und niederer Einkommen, kinderarmer und kinderreicher Arbeitslosen und Beschäftigten (horizontal). ${ }^{11}$

Der Zweck der Umverteilung ist daher ein doppelter. Einerseits sollen Einkommensbezieher durch Leistung eigener Beiträge Einkommen auch für Zeiten des Nichterwerbs gesichert sein und andererseits ist auch Zweck der Umverteilung, die Einkommen der Staatsbürger zugunsten der sogenannten "marktpassiven" tendenziell zu egalisieren, um auf diese Weise einen Lastenausgleich herbeizuführen.

9 Standardsystem, Seite 47.

10 Edgar Nawroth, in : Neue Ordnung, Paderborn 1/1962, Seite 5.

11 Anton Burghardt, Lehrbuch der allgemeinen Sozialpolitik, Berlin 1966. 
Dabei ist aber auch zu berücksichtigen, daß primär Einkommensbezieher in Fällen besonderer Lebenslage, z.B. Krankheit, zu ihren Einkommen noch Transfereinkommen dazu beziehen können.

\section{Die gesetzliche Sozialversicherung als intermediäre Finanzgewalt}

\subsection{Grundsätzliches}

Eine Zusammenschau, ein Einordnen der Einzelkenntnisse bei der heutigen Zersplitterung, Auffächerung und notwendigen Spezialisierung ist wichtiger denn je, um einen Überblick nicht zu verlieren und den derzeitigen Standort zu bestimmen. Die Entwicklung gerade auf dem sozialpolitischen Gebiet geht besonders stürmisch vor sich und zeigt vielfältige Nebenwirkungen auf fast allen Bereichen des Zusammenlebens und damit auch im allgemeinen volkswirtschaftlichen und speziellen finanzwirtschaftlichen Bereich. Aus diesem Grund wurde versucht, den derzeitigen Status gleichsam als Momentaufnahme zu sichten und einzuordnen und zwei Gebiete zusammenzuführen, die viel Gemeinsames aber auch manch Trennendes haben, nämlich die Sozialversicherung und das staatliche Finanzwesen, wobei im Hinblick auf den Umfang dieser Sachgebiete und zur Verdeutlichung der Tatbestände im wesentlichen die Schwerpunkte angerissen werden, wobei nicht übersehen wird, daß so manchem Einzelproblem eine intensivere Befassung zusteht.

\subsection{Interdependenz sozial- und finanzpolitischer Maßnahmen}

Zwischen den verschiedenen Ordnungselementen besteht im Staat eine allgemeine Interdependenz.

Das staatliche Finanzwesen hat selbst weitgehende sozialpolitisch wirkende Aufgaben, da es neben der Korrektur marktwirtschaftlicher Prozesse, insbesondere für die effektivere gerechte Verteilung des volkswirtschaftlichen Ertrages und der staatlichen Hilfsdienste auf Einkommens- oder sonstige soziologisch relevante Gruppen - wobei dies entweder vor oder nach der Abgabeneinhebung sein kann - zu sorgen hat.

Die Sozialversicherung ordnet sich in diesen Aufgabenbereich des staatlichen Finanzwesens systemgerecht ein, da sie einerseits einkommensvermindernd durch Beitragseinhebung und andererseits einkommensbildend bzw. einkommenserhöhend durch Leistungsgewährung in beiden Richtungen des finanzpolitischen Kompetenzbereiches wirkt. Sie ist daher nahezu ein ideales Instrument zur Durchsetzung sozial- und finanzpolitischer Zielbestimmungen. Bedauerlicherweise wurde gerade die allgemeine Interdependenz und das damit eingeschlossene Inzidenzproblem dieser Ordnungen noch sehr wenig untersucht und Recktenwald ${ }^{12}$ stellt dazu fest :

“... und hier erstaunt es immer wieder, wie wenig wir im Grund von der tatsächlichen Belastung und Begünstigung Einzelner und einzelner Gruppen, Zweige und Regionen wissen, obwohl es für viele zum täglichen Brot gehört, über soziale Gerechtigkeit finanzpolitischer Maßnahmen zu urteilen oder zu befinden." Seite 31 .

12 Horst Claus Recktenwald: Die Finanzwissenschaft unserer Zeit, Stuttgart 1965, 
Sicherlich ist das Unterfangen, sowohl mikro- als auch makroökonomisch einigermaßen Klarheit darüber zu gewinnen, inwiefern sich die Lasten und Begünstigungen personell, regional und funktional verteilen, außerordentlich schwer, da die tatsächliche Weiterwälzung der Abgaben und der Fluß der Leistungen im Marktprozeß nur über wenige Stufen $\mathrm{zu}$ verfolgen und $\mathrm{zu}$ messen sind. Doch gerade deswegen ist es umso wichtiger, die Verknüpfungen und Fäden dieses Netzwerkes allmählich aufzudecken und etwas mehr Licht in das Halbdunkel zu bringen, um die soziale Finanzpolitik rationaler, gerechter und dauerhafter in der Effizienz gestalten zu können, denn je mehr Kenntnisse über die Auswirkungen vorliegen, umso leichter wird das geeignete Instrument für gezielte staatliche Maßnahmen ausgewählt werden können.

\subsection{Der strukturelle finanzwirtschaftliche Föderalismus der Sozialversicherung}

Durch den föderalistischen Staatsaufbau - wobei die föderalistische Gewaltenteilung nicht nur in territorialer, sondern auch in struktureller Hinsicht (z.B. in Form der Selbstverwaltung) gesehen werden kann - ergibt sich ein Pluralismus der Finanzgewalten, da die Finanzhoheit für den eigenen Wirtschaftsbereich ein wesentlicher Bestandteil der Autonomie ist. Der pluralistische Charakter des Finanzsystems wird noch verstärkt durch die Übertragung von Akten der Hoheitsverwaltung auf autonome oder halbautonome Organisationen, die mit den ihnen zugewiesenen und/oder von ihnen vereinnahmten Mitteln ein gesondertes Budget zu erstellen haben.

A) Fiskus - Neben(Hilfs)Fiskus ; Sozialversicherung

Solche Organisationen, $\mathrm{zu}$ denen im besonderen die Sozialversicherungsträger zählen, werden, weil sie sich mit ihren Finanzhaushalten wie Zahnräder in den Bundeshaushalt einordnen, als intermediäre Finanzgewalten ${ }^{13}$ bezeichnet. Sie unterstützen den Staat bei der Erfüllung fiskalischer Aufgaben ${ }^{14}$ und werden daher auch vielfach mit "Hilfsfiskus", " hilfsfiskalischen Verzweigungen ", "Parafiskus" oder "Nebenfiskus" angesprochen, während in den USA die Bezeichnungen "government corporation" gebräuchlich ist. In Italien und Frankreich werden sie - bezeichnenderweise für die zentralistische Staatsform - parastaatliche Organismen (organismes para-étatiques) genannt.

Das Zusammenleben vielschichtiger Gruppen im modernen Staat schafft neue kollektive, vor allem soziale Bedürfnisse, die zu einem großen Teil von der Sozialversicherung getragen werden. Um die erforderlichen Mittel für diese nicht territorialen, sondern sozialen und wirtschaftlichen Aufgaben bereitstellen zu können, nimmt der Staat eine Aufgliederung und Trennung seiner Funktionen vor. ${ }^{15}$

Die Funktionsübernahme erfolgt von zwei Seiten und zwar einerseits vom Staat - nach dem Subsidiaritätsprinzip - und andererseits vom einzelnen Staatsbürger, der für sich selbst vorsorgen müßte, zieht sie zusammen und befriedigt sie kollektiv. ${ }^{16}$

13 Fritz Karl Mann : Finanztheorie und Finanzsoziologie, Göttingen 1959, Seite 25.

14 H. Meinhold, Fiskalpolitik durch sozialpolitische Parafisci, Tübingen 1976, Seite 45.

15 Stefani G. : "Voraussetzungen und Entwicklungen der Parafiskalität im Werk Emanuele Morsellis", in : FA, NF, Band 15, 1954/55, Seite 286.

16 Andreae, C. A. : "Die parafiskalischen Gebilde in finanzwirtschaftlicher Schau ", in : Festschrift für Walter Heinrich, Wien 1963, Seite 333. 
Damit wird die Parafiskalität aus dem staatlichen in den intermediären Raum verlegt, dessen Wesen darin besteht, daß eigenständige Gebilde zwischen dem Staat und dem Einzelnen spezifische Gruppenaufgaben wahrnehmen und sich dadurch deutlich vom staatlichen Funktionsbereich abheben. ${ }^{17}$

B) Gründe für die Teilung der Finanzgewalt

Die Gründe für die Teilung der Finanzgewalt liegen teilweise darin, daß z.B. die Sozialversicherung eine größere Spezialisierung und Beweglichkeit gewährleistet und im Rahmen der Selbstverwaltung versichertennaher verwalten kann. Auch die Abgabenmotivierung zur Hinausschiebung des sogenannten "psychological breaking point" stellt einen nicht unbeachtlichen Faktor dar, da mit der gesonderten Einhebung der Sozialversicherungsbeiträge die Gesamtheit der staatlichen Abgaben in kleinere Beträge aufgeteilt wird und außerdem die Sozialversicherungsbeiträge, trotz eines gewissen "Grolleffektes ",18 in Erwartung künftiger persönlich bedeutsamer Leistungen erbracht werden. Aus diesem Grund erscheint der Vorschlag eines einheitlichen Sozialversicherungsbeitrages nicht zweckmäßig. ${ }^{19}$

Sicherlich war es auch erwünscht, gewisse öffentliche Aufgaben unabhängigen Organisationen, die den Privatunternehmungen nachgebildet sind, zu übertragen. Auch spricht eine gewisse Zweckmäßigkeit dafür, den Großteil des Ansatzes "Sozialversicherung " aus dem Bundesbudget herauszunehmen und gesondert zu budgetieren, da ja die Leistungsansätze im wesentlichen durch Gesetze vorgegeben sind und damit zumindest grundsätzlich eine Fixpost darstellen.

Diese Vorgangsweise findet sich bei marktwirtschaftlich orientierten Wirtschaftsverfassungen, da bei diesen das Staatsbudget nur der Finanzrechnung für die Durchführung spezieller öffentlicher Aufgaben, die der parlamentarischen Kontrolle bedürfen, dient. In zentralverwaltungs-wirtschaftlich orientierten Wirtschaftfassungen stellt das Staatsbudget eine gesamtwirtschaftliche Finanzrechnung dar und hat daher vollkommen andere Aufgaben; so erfüllt es unter anderem auch die Funktionen des Kapitalmarktes. Der Staatshaushalt ist daher in diesen Fällen im wesentlichen ident mit dem Haushalt der Volkswirtschaft.20

Auch die sozialpolitische Bedeutung der Sozialversicherung spricht für eine finanzhoheitliche Sonderstellung, damit ein als richtig erkanntes sozialpolitisches Ziel ohne dem Einfluß wechselnder parlamentarischer Mehrheiten oder der Tagespolitik allzu sehr zu unterliegen, über größere Perioden hindurch verfolgt und verwirklicht werden kann. Der Erfolg hat - wie die Entwicklung der Sozialversicherung vom Beginn bis zum heutigen Standard beweist - dieser Form recht gegeben und ist schon allein im Hinblick auf die Größenordnung der Einnahmen und der Ausgaben sowie deren Streuung, finanzpolitisch eine der wichtigsten der intermediären Finanzgewalten geworden.

17 Smekal, C. : Die Finanzwirtschaft intermediärer Gruppen, Innsbruck 1969, Seite 42.

18 Richard A. Musgrave : Finanztheorie, Tubingen 1969, 2. Auflage, Seite 192.

19 Martin Binder: "Gedanken zur Finanzierung der Sozialleistungen ", Versicherungsgrundschau, Nr. 2/1971, Seite 41 ff.

20 Elisabeth-Liefmann-Keil : Einführung in die politische Okonomie, Freiburg-Breisgau 1964, Seite 236. 
C) Gesamtbetrachtung des pluralistischen Finanzwesens

Bei einer solchen Betrachtungsweise der pluralistischen Struktur des modernen Finanzwesens wird es aber auch notwendig, die überlieferten Begriffe wie Gesamtsteuerlast, öffentlichen Gesamtausgaben, Haushaltsgleichgewicht usw. mit neuem Inhalt zu erfüllen. So besteht die Gesamt(" steuer ")last nicht nur aus der Summe aller Staats-, Länder- und Gemeindesteuern, sondern neben anderen Umlagen und Beiträgen im besonderen auch aus den Pflichtversicherungsbeiträgen der Sozialversicherung und den für die Sozialversicherung zweckgebundenen Abgaben (wie z.B. die Abgabe land- und forstwirtschaftlicher Betriebe oder ein Teil des Aufkommens an Gewerbesteuer). Diese haben bereits verbandsmäßige Formen und damit den Charakter von "Gruppensteuern " angenommen, die anknüpfen an die Leistungsfähigkeit der Mitglieder und entsprechend dem Solidaritätsprinzip einen allgemeinen Lasten- und Gefahrenausgleich bringen. ${ }^{21}$

Die vom Staat abgeleitete, gesetzlich verliehene Beitragsautonomie der Sozialversicherungsträger - aber auch der anderen parafiskalischen Verwaltungseinheiten endet unabweislich an der Grenze zur Steuer. ${ }^{22}$

So sind viele gewöhnt, den Staat, weil er über mehr Zwangsmittel verfügt, für stärker zu halten als die Selbstverwaltungen und die Verbände. Wenn ein Problem schwer lösbar erscheint, wird deshalb immer wieder die Forderung erhoben, es durch staatlichen Zwang zu regulieren.

Es zeigt sich jedoch, daß Politiker schwächer sind als Sozialversicherungsfunktionäre. Das ist eine Erfahrung, die nur wenigen in ihr polit-ökonomisches Konzept paßt. Tatsächlich hat sich bisher der Belastungswiderstand gegenüber Steuern als größer erwiesen als gegenüber Sozialversicherungsbeiträgen. Die Individuen haben nämlich das Gefühl, daß sie für ihre Sozialversicherungsbeiträge eine Gegenleistung erhalten, während sie bei den Staatsausgaben glauben, daß diese zwar ihnen zugute kämen, indes aber andere die Steuern dafür zahlen, Beiträge zu bezahlen ist "sozial ", Steuern sind nur dann "sozial", wenn sie andere treffen. ${ }^{23}$

Der Budgetrahmen der Sozialversicherung in Österreich betrug $196410,5 \%$ des Bruttonationalproduktes und 1974 12,5\%; der Anteil am Volkseinkommen umfaßte $196413,8 \%$ und $197416,7 \%$ und gegenüber dem Bundesbudget 1964 38,0\% und 1974 48,4\%. Diese Größenordnungen bestätigen zu Recht die finanzpolitische Bedeutung der Sozialversicherung einerseits und andererseits auch den enormen Mittelbedarf, der zum Großteil eine fiskalische Fixpost darstellt, die mit anderen staatswirtschaftlichen Aufgaben in Einklang zu bringen sind.

\section{Wirkungen der Finanzströme der Sozialversicherung}

Die von der Sozialversicherung hervorgerufenen Finanzströme durch die öffentlichen Haushalte werden von Jahr zu Jahr stärker. Dies beeinflußt den Güterkreislauf

21 K. M. Hettlage : Beiträge, in : Hd. SW, 1. Band, 1956, Seite 729, sowie R. Kulmmer : Beiträge, in : Hb d. FiWi, 2. Band, 2. Auflage, Tubingen 1956, Seite 237.

22 Josef Isensee: Umverteilung durch Sozialversicherungsbeiträge, Berlin 1973, Seite 57.

23 Philipp Herder-Dorneich: "Rationales und Irrationales, Die Gesundheitspolitik braucht eine neue Ökonomie", in : Die Politische Meinung, Heft Nr. 171, Seite 19. 
der Wirtschaft sowohl in der Richtung als auch im Ausmaß wesentlich, ohne daß die fiskalische Handhabung dadurch einfacher geworden wäre und verlagert in der Folge das Problem der Übertragung von Sozialleistungen immer mehr in den Bereich des Ökonomischen. Einnahmen und Ausgaben für die soziale Sicherung haben Größenordnungen erreicht, die es dem Staat gestatten, mit Hilfe der Sozialbilanzen einen wesentlichen Einfluß auf die Gestaltung des Wirtschaftsablaufes nehmen. Unterstützt werden diese im Gewicht der Sozialfinanzen liegenden Möglichkeiten durch die zunehmende Verfeinerung des sozialpolitischen und wirtschaftstheoretischen Denkens, das dem Politiker jene Erkenntnis liefert, die notwendig ist, um die öffentlichen Finanzen im vollen Bewußtsein ihrer ökonomischen Auswirkungen einzusetzen. Die Mittel der Sozialversicherung sind immer mehr in der Lage, die Rolle eines "Stabilisators" im öffentlichen Haushalt einzunehmen. ${ }^{24,} 25$

Werden alle Maßnahmen des Staates für eine einigermaßen gerechte Einkommensverteilung zusammengefaßt, so zeigt sich, daß die Finanzpolitik mitten in die Sozialpolitik hineinreicht und gerade im Rahmen der Umverteilungspolitik einen wesentlichen Bestandteil der Sozialpolitik darstellt. Die Abstimmung zwischen Finanz- und Sozialpolitik sollte so erfolgen, daß sich die funktionellen Rollen bei der Einkommensverteilung zwischen der staatlichen Finanzwirtschaft und der Sozialversicherung mit ihren verschiedenen Trägern gegenseitig ergänzen. Die Mehrgleisigkeit der Umverteilungsmaßnahmen ist sicherlich kompliziert und schwer überschaubar. Zur Erzielung einer größtmöglichen und beständigen Gesamtwirkung müssen die einzelnen Maßnahmen so aufeinander abgestimmt werden, daß die Ziele der Sozialpolitik erreicht werden. So sind die Leistungen der Umverteilungsträger so zu koordinieren, daß menschenwürdige (Mindest)Einkommen zustande kommen und die Beitragspolitik sowie die steuerliche Einkommensredistribution sich darin einordnen. ${ }^{26}$

Es wird daher bei jeder Maßnahmen zu prüfen sein, welche anderen dazu parallel verlaufen oder sich überschneiden oder gar entgegenwirken und dann die Summe der Vorgänge aufeinander abzustimmen, um einen sinnvollen Gesamteffekt zu erreichen.

24 Harmut Hensen : "Die Finanzen der sozialen Sicherung im Kreislauf der Wirtschaft und Versuche einer ökonomischen Analyse" in : Kieler Studien, Forschungsbericht über das Institut für Weltwirtschaft an der Universität Kiel, herausgegeben v. Fritz Baade, Kiel 1955, Seite 9.

25 Vgl. für den Bereich der Pensionsversicherung, Alfred Radner : in " Die Stellung der Pensionisten in der modernen Wirtschafts- und Gesellschaftsordnung ", Versicherungsrundschau $12 / 1972$ und $1 / 1973$, Seite 345 bzw. 9.

26 Heinz Haller, Finanzpolitik, Tübingen-Zürich, 1961. 